\title{
Quantificação de Componentes Monocíclicos da Mancha Angular do Feijoeiro*
}

\author{
Maristella Dalla Pria1, Lilian Amorim² \& Armando Bergamin Filho² \\ 'Departamento de Fitotecnia e Fitossanidade, Universidade Estadual de Ponta Grossa, Praça Santos Andrade s/n, \\ CEP 84010-790, Ponta Grossa, PR, fax: (042)220-3072, email: mdallapria@uepg.br; ${ }^{2}$ Departamento de Entomologia, Fitopatologia \\ e Zoologia Agrícola, Escola Superior de Agricultura “Luiz de Queiroz”, Cx. Postal 09, CEP 13418-900, Piracicaba, SP
}

(Aceito para publicação em 09/04/2003)

Autor para correspondência: Maristella Dalla Pria

DALLA PRIA, M., LILIAN AMORIM \& BERGAMIN FILHO, A. Quantificação de componentes monociclíclos da mancha angular do feijoeiro. Fitopatologia Brasileira 28:394-400. 2003.

\section{RESUMO}

Foram realizados experimentos em ambiente controlado, em câmaras de crescimento, com duas cultivares de feijoeiro (Phaseolus vulgaris), Rosinha e Carioca, para a quantificação de componentes monocíclicos da mancha angular. Os componentes monocíclicos avaliados foram: período de incubação, severidade da doença e taxa de crescimento das lesões, sob as temperaturas de 6, 10, 14, 17, 21, 25,29 e $33^{\circ} \mathrm{C}$. Nenhum sintoma da mancha angular ocorreu em $6 \mathrm{e}$ $33{ }^{\circ} \mathrm{C}$. A severidade da mancha angular foi elevada na faixa de temperatura entre 20 e $25^{\circ} \mathrm{C}$ e o período de incubação foi favorecido pela temperatura de $25^{\circ} \mathrm{C}$, para as duas cultivares testadas. Esta temperatura também proporcionou a maior taxa de crescimento das lesões e estes dados foram ajustados, em função da temperatura por uma equação do primeiro grau. $\mathrm{O}$ efeito do período de molhamento foliar $(5,6,12,18,24,30,36,42,48$ e $54 \mathrm{~h})$ na severidade da doença também foi avaliado. A severidade da mancha angular do feijoeiro aumentou com o aumento da duração do molhamento foliar até o máximo de $54 \mathrm{~h}$. As variações de severidade da mancha angular do feijoeiro, em relação ao número de horas de molhamento foliar, foram explicadas pelo modelo de Richards. A função Beta-Richards explicou o efeito combinado da temperatura e da duração do molhamento foliar na severidade da mancha angular do feijoeiro para ambas as cultivares.

Palavras-chave adicionais: Phaseolus vulgaris, temperatura, duração molhamento foliar.

\section{ABSTRACT}

Quantification of monocyclic components of the angular leaf spot of common bean

Monocyclic components of angular leaf spot, were quantified on two bean (Phaseolus vulgaris) cultivars Rosinha and Carioca in growth chambers. Incubation period, disease severity and lesion growth rate were assessed, at temperatures of 6, 10, 14, 17, 21, 25, 29 and $33{ }^{\circ} \mathrm{C}$. No angular leaf spot symptoms occurred at 6 and $29{ }^{\circ} \mathrm{C}$. Severity for angular leaf spot was high between temperatures of 20 and $25{ }^{\circ} \mathrm{C}$, and the incubation period for both varieties tested was favoured at a temperature of $25{ }^{\circ} \mathrm{C}$. This temperature also provided the greatest lesion growth rate and these data were adjusted by a first grade equation in function of the temperature. The effect of the leaf wetness duration $(5,6,12,18,24,30,36,42,48$ and $54 \mathrm{~h})$ in the severity of disease symptoms was studied as well. Severity for the angular leaf spot increased with the wetness duration period to the maximum of $54 \mathrm{~h}$ tested. The variations of angular leaf spot in relation to the number of hours of leaf wetness were explained by the Richards model. The Beta-Richards function explained the combined effects of the temperature and leaf wetness duration in the severity of angular leaf spot, for both varieties.

\section{INTRODUÇÃO}

A mancha angular do feijoeiro (Phaseolus vulgaris L.) causada por Phaeoisariopsis griseola (Sacc.) Ferraris, Sin. Isariopsis griseola (Sacc.), está amplamente distribuída e pode causar sérios danos à cultura (Barros et al., 1958; Singh \& Saini, 1980). Durante muito tempo essa doença foi considerada secundária devido a seu aparecimento tardio nas plantações e por causar poucos danos à cultura em termos de produção (Paradela Filho, 1972; Viera, 1974). No entanto, a partir dos últimos anos, passou a ser considerada entre as principais

\footnotetext{
*Parte da tese de doutorado do primeiro autor, ESALQ/USP (1997), com financiamento do $\mathrm{CNPq}$.
}

doenças desta leguminosa, sendo a ela atribuídas as perdas de muitas lavouras. As razões para essa mudança são ainda desconhecidas, acreditando-se que o plantio de materiais suscetíveis aliados a um ambiente favorável tenham proporcionado condições ideais ao seu desenvolvimento (Sartorato, 1989).

A fonte de inóculo primário do patógeno inclui sementes contaminadas e restos de plantas infetadas. Em ambos os casos, os esporos produzidos são disseminados para as folhas pela ação do vento, respingos de chuva, ou ambos. Os conídios germinam na superfície foliar, e as hifas penetram através dos estômatos crescendo entre as células do parênquima foliar. Aproximadamente nove dias após a infecção, o patógeno coloniza extensivamente o parênquima foliar formando lesões 
Quantificação de componentes monocíclicos da mancha angular (Phaeoisariopsis griseola)...

necróticas. A esporulação ocorre em condições de alta umidade. No campo, a doença é favorecida por temperaturas entre $16 \mathrm{e}$ $28^{\circ} \mathrm{C}$, o desenvolvimento máximo ocorre a $24^{\circ} \mathrm{C}$ e a infecção é limitada a $32{ }^{\circ} \mathrm{C}$ (Cardona-Alvarez \& Walker, 1956). Importante também para o desenvolvimento de epidemias são eríodos de alta umidade relativa, suficientemente longos (24 a $48 \mathrm{~h}$ ), alternados por períodos de baixa umidade e ação de ventos. Além desses fatores, o desenvolvimento de epidemias é influenciado pelo sistema de cultivo. Plantas inoculadas mantidas em câmara úmida por no mínimo $3 \mathrm{~h}$ tornam-se infetadas. No entanto, um aumento na duração do período de câmara úmida para $24 \mathrm{~h}$ proporciona um aumento médio de 20 vezes no número de lesões por planta (Kulik, 1984).

Trabalhos realizados em ambiente controlado, em câmaras de crescimento, fornecem uma sólida base para o melhor entendimento do efeito dos fatores ambientais no desenvolvimento das epidemias (Kranz \& Hau, 1980). Dados obtidos em câmaras de crescimento fornecem uma idéia do que possivelmente ocorre no campo, mas podem não simular com exatidão o desenvolvimento da doença na natureza. Assim, da mesma forma que um modelo é uma representação simplificada da realidade, o ambiente controlado é uma representação simplificada do ambiente de cultivo (Rotem, 1988).

A influência da temperatura na quantificação dos componentes monocíclicos como período latente, frequiência de infecção, período infeccioso e produção de esporos tem sido estudada em diversos sistemas patógeno-hospedeiro, já que esses dados são de grande utilidade na construção de modelos de simulação de epidemias (Zadoks \& Schein, 1979). No entanto, para o sistema $P$. griseola-feijoeiro, há poucos dados sobre a influência da temperatura nos componentes monocíclicos.

O período de molhamento refere-se ao período de tempo durante o qual a parte aérea da planta permanece molhada (Sutton et al., 1984). A ocorrência de água sobre a superfície das plantas constitui-se em importante fator no desenvolvimento de doenças. A importância relativa do orvalho para o período de molhamento é acentuada, principalmente quando a precipitação não é freqüente e a quantidade de água livre permanece sobre as superfícies vegetais durante um tempo relativamente curto (Crowe et al., 1978). Fundamentalmente, a importância do orvalho relaciona-se à ocorrência de doenças de plantas e a avaliação do período de molhamento constituise num meio de determinação do potencial de infecção de certas doenças (Howard \& Gillespie, 1985). Vários sistemas de alerta e de previsão de doenças de plantas utilizam a duração do período de molhamento, entre outras variáveis, para estabelecer os níveis de severidade de doenças, a exemplo do míldio da videira (Vitis vinifera L.) causado por Plasmopara viticola (Berk \& Curtis) Berl \& de Toni, da requeima da batata (Solanum tuberosum L.) causada por Phytophthora infestans (Mont) de Bary, da sarna da macieira (Malus dometica Borkh) causada por Venturia inaequalis (Cooke) Winter e da requeima do tomateiro (Lycopersicon esculentum Mill) causada por Phytophthora infestans (Mont) de Bary (Pennypaker \& Stevenson, 1982).
O objetivo deste trabalho foi avaliar o efeito da temperatura e do período de molhamento sobre os componentes monocíclicos da mancha angular, em plantas de feijoeiro mantidas sob condições de ambiente controlado.

\section{MATERIAL EMÉTODOS}

\section{Avaliação do efeito da temperatura nos componentes monocíclicos da mancha angular}

Utilizaram-se duas cultivares de feijão, Rosinha (altamente suscetível à mancha angular) e Carioca (moderadamente suscetível à mancha angular) (Rava \& Sartorato, 1994). As duas cultivares foram semeadas em vasos de alumínio contendo 2,5 1 de solo esterilizado, mantidos em casa de vegetação. Cinco dias após a emergência das plântulas, foi realizado o desbaste mantendo-se duas plantas por vaso. Quarenta e oito horas antes da inoculação, os vasos foram transferidos para câmaras de crescimento (marca CONVIRON modelo E7), com temperatura e fotoperíodo previamente regulados, para aclimatação das plantas.

O fungo $P$. griseola foi multiplicado em placas de Petri com meio de suco de tomate (MSTO) para multiplicação e produção de inóculo (Dalla Pria et al., 1997). A suspensão de conídios de $P$. griseola foi obtida de culturas incubadas por um período de 20 dias, mantidas a $22{ }^{\circ} \mathrm{C} \pm 2$, no escuro. As placas com as colônias fúngicas foram lavadas com água destilada, acrescida de Tween 20 (1 gota para cada $100 \mathrm{ml}$ ), com o auxílio de um pincel de cerdas macias, para auxiliar na liberação dos conídios. A suspensão de esporos obtida foi calibrada para $1,5 \times 10^{5}$ conídios $/ \mathrm{ml}$, pré-estabelecida por meio de testes preliminares.

As plantas foram inoculadas quando a primeira folha trifoliolada encontrava-se completamente expandida, aproximadamente 20 dias após a semeadura. A superfície superior e a inferior das duas primeiras folhas trifolioladas foram atomizadas com a suspensão de inóculo até o ponto de escorrimento. Após a inoculação, as plantas permaneceram em câmara úmida (armação de arame e cobertura de saco plástico umedecido, em cada vaso), dentro de câmaras de crescimento, com fotoperíodo de $12 \mathrm{~h}$ de luz. Plantas do tratamento testemunha foram atomizadas com água destilada + Tween 20 (1 gota para cada $100 \mathrm{ml}$ de água). As temperaturas avaliadas nesse ensaio foram: $6,10,14,17,21,25,29$ e $33^{\circ} \mathrm{C}$, e o período de molhamento foliar utilizado foi $48 \mathrm{~h}$ (Bassanezi et al., 1998). O delineamento experimental foi inteiramente casualizado, com quatro repetições. Cada parcela experimental era constituída de um vaso, com duas plantas, onde duas folhas trifolioladas por planta foram avaliadas.

Foram avaliadas as seguintes variáveis: tamanho médio das manchas, por meio de escala de tamanho de lesões (Bassanezi, 1995); severidade (porcentagem de tecido foliar atacado) da doença (Godoy et al., 1997); freqüência de infecção (número de lesões $/ \mathrm{mm}^{2}$ ).

Com a média dos dados de severidade para cada uma das temperaturas testadas foram estabelecidas as curvas de progresso da doença, considerando-se a porcentagem de 
tecido doente em função de dias após a inoculação. As curvas foram ajustadas através de regressão não-linear utilizando-se o modelo de Richards:

$$
\begin{aligned}
& \left(Y=K(1-(\beta) E X P(-R X))^{(1 /(1-M)}\right), \text { quando } M<1 \\
& \left(Y=K(1+(\beta) \operatorname{EXP}(-R X))^{(1 /(1-M)}\right), \text { quando } M>1
\end{aligned}
$$

onde $\mathrm{Y}$ é a severidade da doença, X é o tempo, K representa a assíntota do modelo, $\beta$ representa a constante de integração, $\mathrm{R}$ a taxa de progresso da doença e $\mathrm{M}$ é o parâmetro que representa a forma da curva (Schuh \& Adamowicz, 1993). Para o ajuste dos dados foi utilizado o programa STATISTICA for Windows versão 4.5 (StatSoft, Tulsa, EUA). Com os valores das assíntotas (K) foi possível obter um gráfico da severidade da mancha angular em função das temperaturas. Os pontos foram ajustados por meio de regressão não-linear, usando-se a função Beta generalizada (Hau \& Kranz, 1990), onde

$$
\mathrm{Y}=\mathrm{B}_{1}\left(\left(\mathrm{X}-\mathrm{B}_{2}\right)^{\mathrm{B} 4}\right)\left(\left(\mathrm{B}_{3}-\mathrm{X}\right)^{\mathrm{B} 5}\right)
$$

os parâmetros $\mathrm{B}_{2}$ e $\mathrm{B}_{3}$ representam as temperaturas mínima e máxima, respectivamente, $\mathrm{Y}$ representa a severidade, $\mathrm{X}$ representa a temperatura, os parâmetros $\mathrm{B}_{1}, \mathrm{~B}_{4}$ e $\mathrm{B}_{5}$ são parâmetros do próprio modelo e não possuem significado biológico.

Ainda com base nas equações ajustadas para cada uma das temperaturas testadas foi possível calcular o período de incubação. O período de incubação (horas) foi considerado como o período de tempo compreendido entre a inoculação e o aparecimento de 50\% dos sintomas (Parlevliet, 1975), isto é, o tempo para que a severidade se iguale a $\mathrm{K} / 2$, estimada para cada uma das temperaturas testadas por meio do modelo de Richards.

Com os dados referentes ao tamanho das manchas para cada uma das temperaturas testadas, foram construídas curvas de progresso da doença. O ajuste dos dados também foi obtido pelo modelo de Richards. Os valores de R (taxa de aumento) não podem ter seus valores comparados diretamente caso tenham sido estimados com diferentes valores do parâmetro M, responsável pela forma da curva (Campbell \& Madden, 1990; Bergamin Filho \& Amorim, 1996). Como os valores do parâmetro $\mathrm{M}$ obtidos nas equações apresentaram pequenas diferenças, foi calculada a média deste parâmetro. $\mathrm{O}$ valor médio encontrado foi fixado e utilizado para recalcular as curvas de progresso da doença para cada uma das temperaturas obtendose novos valores para cada um dos parâmetros do modelo de Richards. Com os novos valores obtidos para o parâmetro R, foi possível construir um gráfico onde se obteve a taxa de crescimento das lesões em $\mathrm{mm}^{2}$ por hora em função de cada temperatura. Os pontos foram ajustados por meio de equação do primeiro grau $(\mathrm{Y}=\mathrm{a}+\mathrm{bX})$, onde $\mathrm{Y}$ representa a taxa de crescimento das lesões $\left(\mathrm{mm}^{2} /\right.$ hora $)$ e X representa a temperatura $\left({ }^{\circ} \mathrm{C}\right)$.

\section{Avaliação do efeito do período de molhamento foliar nos componentes monocíclicos da mancha angular}

Após o estabelecimento da temperatura ótima para o desenvolvimento das doenças, foram realizados experimentos para determinação do efeito da duração do período de molhamento foliar sobre os componentes monocíclicos da mancha angular do feijoeiro. Os períodos de câmara úmida testados foram: 5, 6, 12, 18, 24, 30, 36, 42, 48 e 54 h, sob temperatura constante de $21^{\circ} \mathrm{C}$. O preparo das mudas, obtenção do inóculo, inoculação e avaliação foram os mesmos utilizados nos experimentos descritos no item anterior. O delineamento experimental foi inteiramente casualizado, com quatro repetições. Cada parcela experimental era constituída de um vaso, com duas plantas, onde foram avaliadas duas folhas trifolioladas por planta.

Avaliou-se a severidade da mancha angular por meio de escala diagramática (Godoy et al., 1997). As observações foram realizadas diariamente até o surgimento dos primeiros sintomas, a partir daí, as avaliações seguiram a intervalos de 12 ou $24 \mathrm{~h}$, dependendo da velocidade de crescimento da doença, e finalizaram quando a porcentagem de tecido atacado não apresentava aumento.

Com a média dos dados de severidade para cada um dos períodos de molhamento foliar testados foram estabelecidas as curvas de progresso da doença, considerando-se a porcentagem de tecido doente em função do tempo. As curvas foram ajustadas por meio de regressão não-linear, utilizando-se o modelo de Richards. Com os dados obtidos foi possível elaborar um gráfico da severidade das doenças em função da duração do molhamento foliar.

O efeito combinado da temperatura e da duração do molhamento foliar na severidade das doenças foi descrito pelo modelo Beta-Richards:

$$
\mathrm{Z}=\mathrm{B}_{1}\left(\left(\mathrm{X}-\mathrm{B}_{2}\right)^{\mathrm{B} 4}\right)\left(\left(\mathrm{B}_{3}-\mathrm{X}\right)^{\mathrm{B} 5}\left(1 \pm(\beta) \mathrm{EXP}\left(-\mathrm{B}_{6} \mathrm{Y}\right)\right)^{\mathrm{B} 7}\right.
$$

e possibilitou o cálculo da superfície resposta. Os parâmetros foram ajustados por meio de regressão não-linear dos dados, onde: $\mathrm{Z}=$ severidade da doença $(\%), \mathrm{X}=$ temperatura $\left({ }^{\circ} \mathrm{C}\right), \mathrm{Y}=$ duração do molhamento foliar (horas), $\beta=$ constante de integração, $\mathrm{B}_{2}=$ temperatura mínima, $\mathrm{B}_{3}=$ temperatura máxima, $\mathrm{B}_{7}=$ forma da curva, $\mathrm{B}_{1}, \mathrm{~B}_{4}, \mathrm{~B}_{5}$ e $\mathrm{B}_{6}=$ parâmetros do modelo.

\section{RESULTADOSE DISCUSSÃO}

\section{Avaliação do efeito da temperatura nos componentes monocíclicos da mancha angular}

$\mathrm{O}$ efeito da temperatura na severidade da mancha angular do feijoeiro pode ser observado na Figura 1. As equações que apresentaram melhor ajuste aos dados de severidade da mancha angular em função da temperatura, com os respectivos coeficientes de determinação, encontram-se na Tabela 1.

Com estes resultados verificou-se que tanto a severidade (Figura 1), quanto o período de incubação (Figura 2), foram sensivelmente afetados pela temperatura. A máxima severidade ocorreu na faixa entre 20 e $25^{\circ} \mathrm{C}$ e o menor período de incubação a $25{ }^{\circ} \mathrm{C}$, para as duas cultivares, resultado semelhante ao obtido por Llanos (1957), em que a severidade 
Quantificação de componentes monocíclicos da mancha angular (Phaeoisariopsis griseola)...

da doença e a desfolha ocorreram com maior intensidade em plantas mantidas a $24^{\circ} \mathrm{Ce} 30^{\circ} \mathrm{C}$. Temperaturas abaixo e acima desta faixa atrasaram o desenvolvimento da doença e, consequentemente, prolongaram o período de incubação. Nas temperaturas de 6 e superiores a $29^{\circ} \mathrm{C}$ não foram observados quaisquer sintomas da mancha angular tanto na cv. Carioca quanto na cv. Rosinha.

Entre as cultivares, observou-se uma ligeira diferença, sendo a severidade levemente maior na cv. Rosinha (Figura 1B) que na cv. Carioca (Figura 1A). Como a severidade é dependente do tamanho de lesões e da frequiência de infecção, já era esperado que houvesse diferença entre as cultivares Rosinha (altamente suscetível) e Carioca (moderadamente suscetível). Verificou-se que também para a mancha angular a temperatura exerce efeito significativo em todas as fases do ciclo das relações patógeno-hospedeiro. Os dados obtidos neste trabalho foram semelhantes aos resultados de Bassanezi et al. (1998), que verificaram comportamento diferente entre as cultivares testadas, Carioca e Rosinha, quanto à severidade máxima observada (Tabela 1).

Tanto os resultados obtidos nestes experimentos com P. griseola quanto os obtidos por Bassanezi et al. (1998), mostraram que a taxa de crescimento das lesões foi favorecida pela temperatura de $24{ }^{\circ} \mathrm{C}$. Conforme resultados obtidos no presente trabalho, a temperatura também influenciou todos os componentes estudados e esta influência foi bem descrita pela função Beta, sendo possível observar o efeito da temperatura

$\mathrm{SEV}=(1,84) *((\mathrm{~T}-(5,75)) * *(0,74)) *(((29,0)-\mathrm{T}) * *(0,37))$
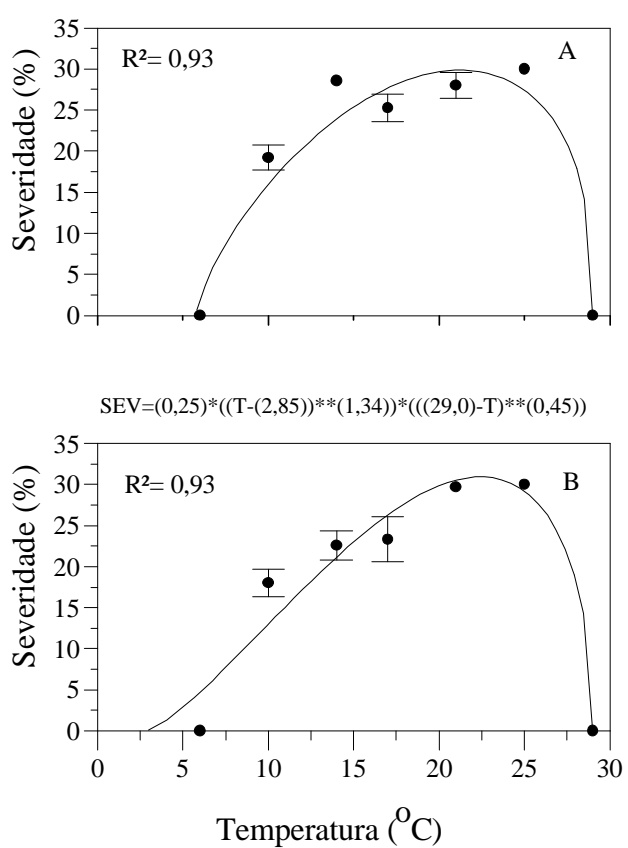

FIG.. 1 - Severidade da mancha angular do feijoeiro (Phaseolus vulgaris) causada por Phaeoisariopsis griseola, em diferentes temperaturas, nas cultivares Carioca (A) e Rosinha (B). Cada ponto representa a média de quatro repetições. Barras representam erro padrão da média. Linhas representam o ajuste do modelo Beta generalizado.
TABELA 1 - Equações e respectivos coeficientes de determinação $\left(\mathrm{R}^{2}\right)$, obtidos nas regressões não-lineares, para ajuste dos dados de severidade da mancha angular do feijoeiro (Phaseolus vulgaris) causada por Phaeoisariopsis griseola, em função do tempo em cada uma das temperaturas, para as cultivares Carioca e Rosinha

\begin{tabular}{|c|c|}
\hline Equação & $\mathbf{R}^{2}$ \\
\hline \multicolumn{2}{|l|}{ cultivar Carioca } \\
\hline $\left.\left.\mathrm{SEV}_{10}{ }^{\mathrm{o}} \mathrm{C}=(22,15)(1-(2,43) \exp (-(0,017) \mathrm{T}))^{(1 /(1-(0,9997)}\right)\right)$ & 0,998 \\
\hline $\left.\left.\mathrm{SEV}_{14}{ }^{\circ} \mathrm{C}=(21,98)(1-(3,16) \exp (-(0,023) \mathrm{T}))^{(1 /(1-(0,9990)}\right)\right)$ & 0,990 \\
\hline $\left.\left.\mathrm{SEV}_{17}{ }^{\circ} \mathrm{C}=(23,96)(1-(0,71) \exp (-(0,023) \mathrm{T}))^{(1 /(1-(0,9993)}\right)\right)$ & 0,992 \\
\hline $\left.\left.\mathrm{SEV}_{21}{ }^{\circ} \mathrm{C}=(28,23)(1-(5,62) \exp (-(0,030) \mathrm{T}))^{(1 /(1-(0,9972)}\right)\right)$ & 0,999 \\
\hline $\left.\left.\mathrm{SEV}_{25}{ }^{\circ} \mathrm{C}=(30,34)(1-(1,71) \exp (-(0,038) \mathrm{T}))^{(1 /(1-(0,9997)}\right)\right)$ & 0,992 \\
\hline \multicolumn{2}{|l|}{ cultivar Rosinha } \\
\hline $\left.\left.\mathrm{SEV}_{10}{ }^{\mathrm{o}} \mathrm{C}=(26,63)\left(1-(3,74) \exp \left(-(0,011)^{*} \mathrm{~T}\right)\right)^{(1 /(1-(0,9899)}\right)\right)$ & 0,990 \\
\hline $\left.\left.\mathrm{SEV}_{14}{ }^{\circ} \mathrm{C}=(23,35)\left(1-(6,38) \exp \left(-(0,020)^{* T}\right)\right)^{(1 /(1-(0,9961)}\right)\right)$ & 0,994 \\
\hline $\left.\left.\mathrm{SEV}_{17}{ }^{\circ} \mathrm{C}=(23,94)\left(1-(2,24) \exp \left(-(0,026)^{* T}\right)\right)^{(1 /(1-(0,9989)}\right)\right)$ & 0,993 \\
\hline $\left.\left.\mathrm{SEV}_{21}{ }^{\circ} \mathrm{C}=(29,99)(1-(4,35) \exp (-(0,037) * \mathrm{~T}))^{(1 /(1-(0,9996)}\right)\right)$ & 0,998 \\
\hline $\left.\left.\mathrm{SEV}_{25}{ }^{\circ} \mathrm{C}=(30,24)(1-(5,85) \exp (-(0,041) * \mathrm{~T}))^{(1 /(1-(0,9992)}\right)\right)$ & 0,990 \\
\hline
\end{tabular}

$\mathrm{Sev}=$ severidade, $\mathrm{T}=$ tempo (horas)

em todas as fases do ciclo das relações patógeno-hospedeiro. A função Beta, que está entre as funções citadas por Hau et al. (1985) e Hau \& Kranz (1990) para explicar o efeito da temperatura sobre o desenvolvimento de doenças de plantas, ajustou-se também aos dados de período de incubação.

Os dados obtidos no presente trabalho concordaram com os dados obtidos in vitro por Cardona-Alvarez \& Walker (1956) e Campos \& Zak (1980). Estes autores mostraram que o fungo $P$. griseola cresce em meio de cultura a partir de $8{ }^{\circ} \mathrm{C}$, incrementa o crescimento com a elevação da temperatura a 24 ${ }^{\circ} \mathrm{C}$ e decresce abruptamente a $28^{\circ} \mathrm{C}$. Nenhum crescimento foi

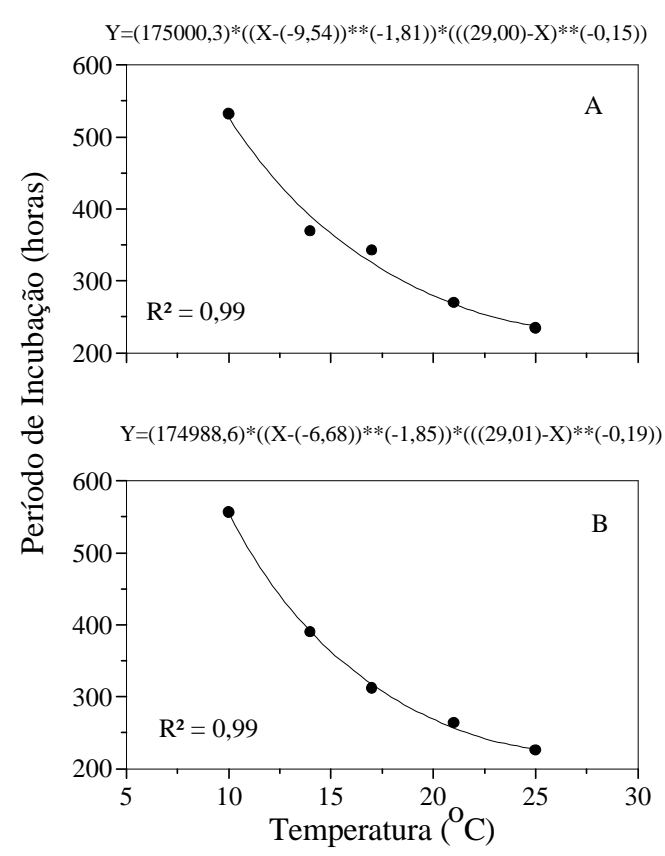

FIG. 2 - Efeito da temperatura no período de incubação da mancha angular do feijoeiro (Phaseolus vulgaris) causada por Phaeoisariopsis griseola, em diferentes temperaturas, nas cultivares Carioca (A) e Rosinha (B). Cada ponto representa a média de quatro repetições. 
observado em temperaturas superiores a $30^{\circ} \mathrm{C}$.

Em todas as temperaturas testadas, a que proporcionou o menor período de incubação foi $25^{\circ} \mathrm{C}$ (Figura 2) e os primeiros sintomas da mancha angular surgiram oito dias após a inoculação, semelhante aos relatos de Llanos (1957), Olave (1958), Campos \& Zak (1980) e Sartorato \& Rava (1994). A $25^{\circ} \mathrm{C}$ o período de incubação encontrado por esses autores foi ao redor de dez e 9,5 dias para as cultivares Carioca e Rosinha, respectivamente. Olave (1958) também verificou que os primeiros sintomas apareceram oito dias após a inoculação quando as plantas foram mantidas a $24,5^{\circ} \mathrm{C}$, com aproximadamente $100 \%$ de umidade relativa, porém Campos \& Zak (1980) observaram o aparecimento dos sintomas aos 12 dias após a inoculação.

De acordo com o efeito da temperatura sobre a taxa de crescimento das lesões (Figura 3), os dados de crescimento das lesões foram melhor ajustados pela equação do primeiro grau, com coeficiente de determinação de 0,95 para as duas cultivares testadas.

A taxa de crescimento das lesões aumentou até atingir um valor máximo, à medida que a temperatura se aproxima do ponto ótimo para o desenvolvimento da doença, em torno de $25{ }^{\circ} \mathrm{C}$, pois no caso da mancha angular do feijoeiro, o aumento da severidade está relacionado com o crescimento das lesões. Como conseqüência do aumento na taxa de crescimento das lesões, houve rápida coalescência das mesmas. As taxas de crescimento de lesões foram semelhantes para as duas cultivares testadas, sendo, portanto, a velocidade de colonização dos tecidos pelo fungo semelhante nas duas variedades.

\section{Avaliação do efeito do molhamento nos componentes monocíclicos da mancha angular}

Períodos de molhamento superiores a $6 \mathrm{~h}$ foram requeridos para o início do processo de infecção (Figura 4). A severidade da mancha angular foi altamente influenciada pelo período de molhamento, sendo estas duas variáveis positivamente correlacionadas, pois o aumento na duração do período de molhamento foliar proporcionou aumento na proporção de tecido foliar atacado, para a cv. Carioca, até $54 \mathrm{~h}$ de molhamento foliar (Figura 4A). O efeito do período de molhamento foi explicado pelo modelo de Richards, modelo este que ajustou bem às curvas com formato sigmóide, com coeficiente de determinação de 0,91 e 0,93 para as cultivares Carioca e Rosinha, respectivamente (Figura 4A e 4B).

Vários pesquisadores têm tentado descrever o processo de infecção em função da temperatura e duração do molhamento foliar. Modelos polinomiais geralmente são usados para descrever o efeito de fatores do ambiente na infecção (Arauz $\&$ Sutton, 1989). Estes modelos são preferidos devido à flexibilidade da curva, dada pelo grau do polinômio. As funções são matematicamente simples e os métodos utilizados para o ajuste das curvas estão disponíveis na literatura (Steel \& Torrie, 1980). Entretanto, funções polinomiais têm pouco significado biológico e o ajuste é limitado pelo grau do polinômio que pode ser usado (Venus \& Causton, 1979). Já o modelo de Richards, baseado em curvas que descrevem processos bioló-

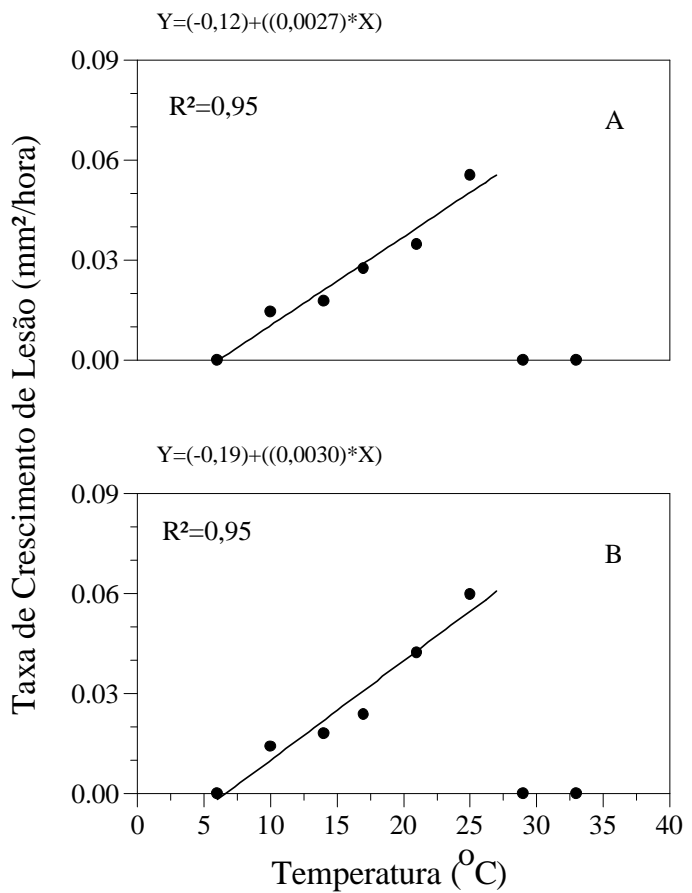

FIG. 3 - Taxa de crescimento das lesões $\left(\mathrm{mm}^{2} /\right.$ hora $)$ da mancha angular do feijoeiro (Phaseolus vulgaris) causada por Phaeoisariopsis griseola, em diferentes temperaturas, nas cultivares Carioca (A) e Rosinha (B).

$\mathrm{SEV}=(29,98) *(1+(5,45) * \operatorname{EXP}(-(0,20) * \mathrm{MOL}))^{* *}(1 /(1-(1,025)))$
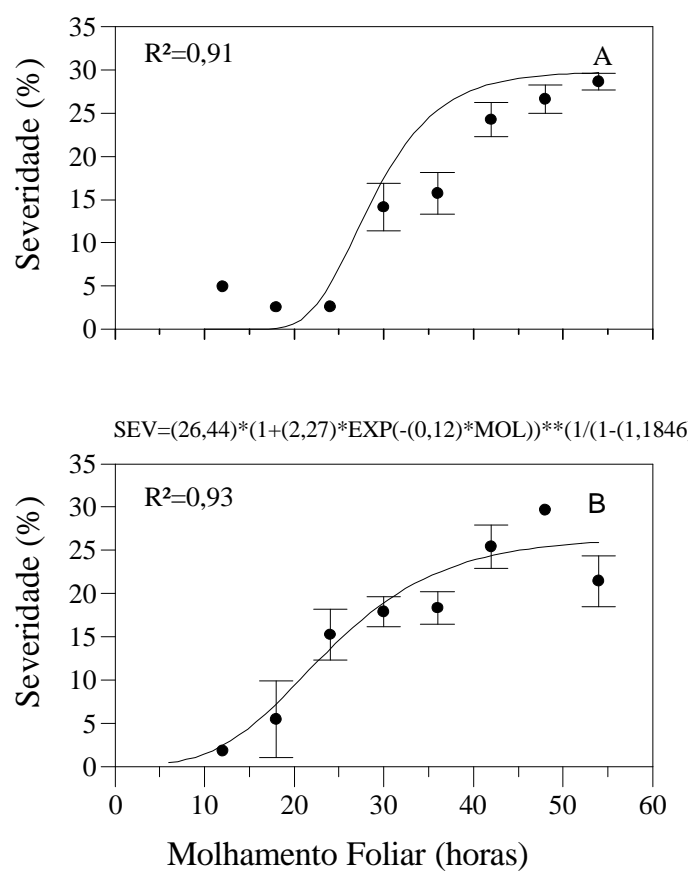

FIG 4 - Severidade da mancha angular do feijoeiro (Phaseolus vulgaris) causada por Phaeoisariopsis griseola, em diferentes períodos de molhamento foliar, nas cultivares Carioca (A) e Rosinha (B). Cada ponto representa a média de quatro repetições. Barras representam erro padrão da média. 
gicos e os parâmetros $\mathrm{K}$, R e M, descrevem completamente a forma da curva. No entanto, a função é mais difícil de ser utilizada e a estimativa direta dos parâmetros nem sempre é possível, particularmente quando os parâmetros estão altamente correlacionados.

Curvas do tipo sigmóide foram também obtidas por Carisse \& Kushalappa (1990), quando estudaram o efeito do período de molhamento sobre o número de lesões de Cercospora carotae (Pass.) Solhein em cenoura (Daucus carotae L.), sendo esta relação bem explicada pelo modelo de Richards. A influência do período de molhamento sobre a máxima infecção de plantas de trigo (Triticum aestivum L.) por Puccinia recondita Rob. Ex Desm. f. sp. tritici e Puccinia striiformis West. também foi descrita pelo modelo de Richards, com parâmetros dependentes da temperatura (Butler \& Jadhav, 1991; Vallavieille-Pope et al., 1995).

Combinando-se as equações obtidas para os dados de temperaturas e duração do molhamento foliar, foi possível calcular a superfície resposta de severidade em função dessas duas variáveis (Figuras 5 e 6). Os dados foram ajustados pelo modelo Beta-Richards, com coeficiente de determinação de 0,92 para a cv. Carioca (Figura 5) e 0,96 para a cv. Rosinha (Figura 6), gerando, respectivamente, as equações $\mathrm{z}=2,15$ ( $\mathrm{x}-$ $6)^{0,69}(20-x)^{0,36}\left((1+21,91 \exp (-0,28 y))^{-73}\right.$ e $z=1,21(x-6)^{0,9}(29$ $\mathrm{x})^{0,43}\left((1+1,27 \exp (-0,081 \mathrm{y}))^{-5,83}\right)$, onde $\mathrm{z}$ é a severidade, $\mathrm{x}, \mathrm{a}$ temperatura e y, a duração do molhamento foliar.

A severidade da mancha angular do feijoeiro aumentou à medida que houve aumento no período de molhamento foliar até $54 \mathrm{~h}$, e a temperatura, com o máximo entre 20 e $25^{\circ} \mathrm{C}$ para a cv. Carioca e para a cv. Rosinha (Figuras 5 e 6 ).

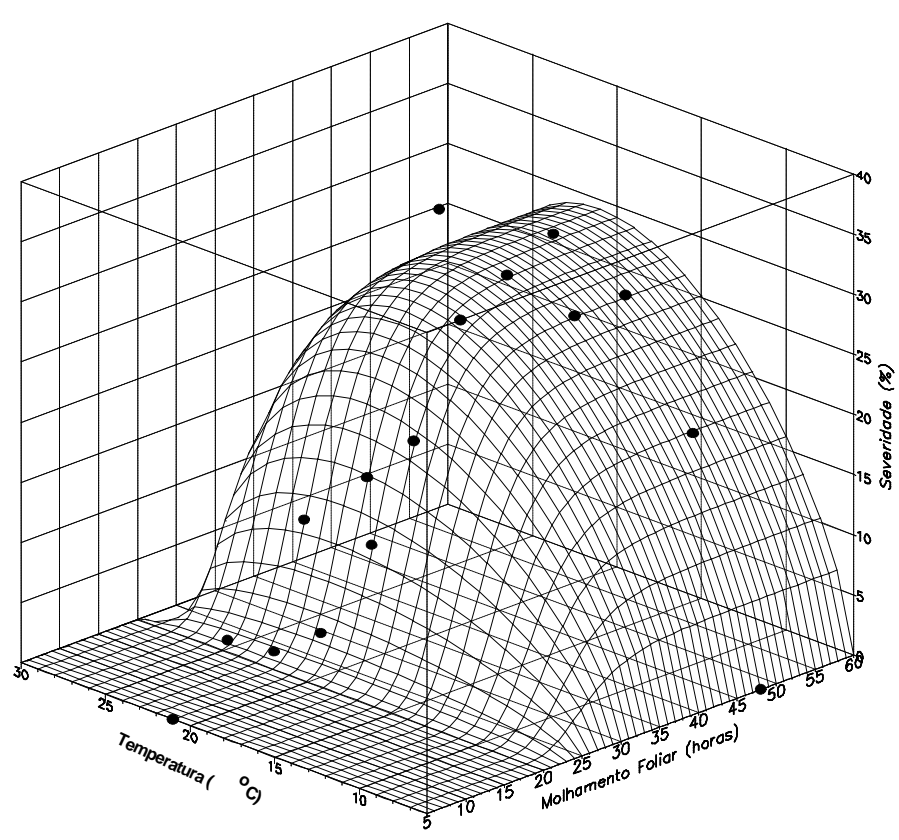

FIG. 5 - Efeito do período de molhamento foliar e da temperatura na severidade da mancha angular do feijoeiro (Phaseolus vulgaris) causada por Phaeoisariopsis griseola, na cultivar Carioca.

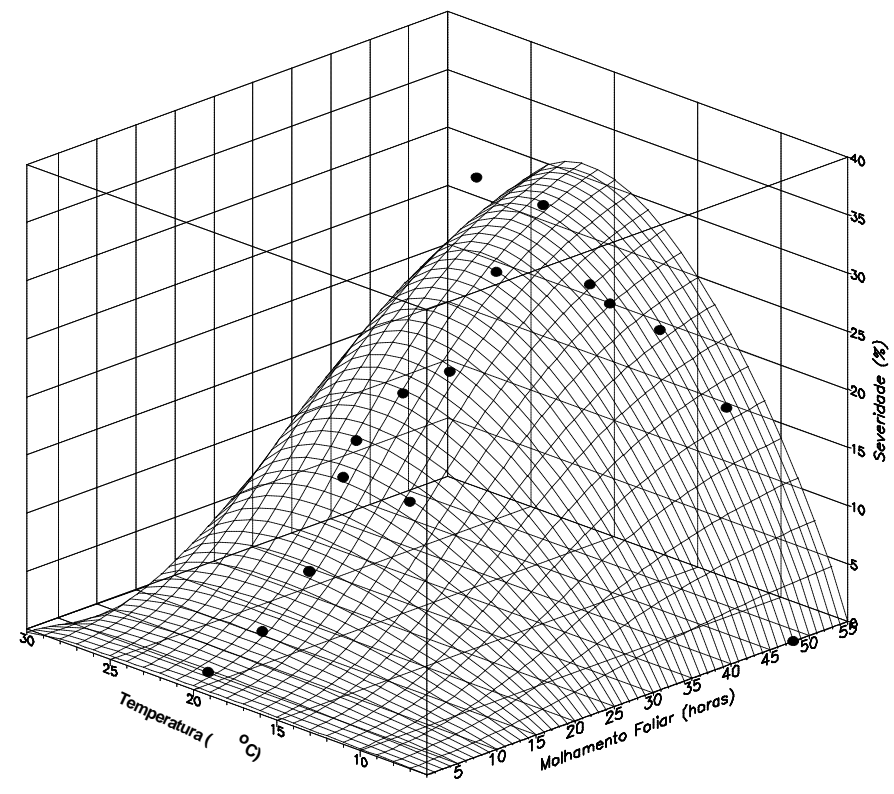

FIG. 6 - Efeito do período de molhamento foliar e da temperatura na severidade da mancha angular do feijoeiro (Phaseolus vulgaris) causada por Phaeoisariopsis griseola, na cultivar Rosinha.

\section{REFERÊNCIAS BIBLIOGRÁFICAS}

ARAUZ, L.F. \& SUTTON, T.B. Temperature and wetness requirements for apple infection by Botryosphaeria obtusa. Phytopathology 79:440-444. 1989.

BARROS, O., CARDONA, C., CARDENOSA, R. \& SKILES, R.L. Angular leaf spot of bean in Colombia. Plant Disease Reporter 42:420424. 1958.

BASSANEZI, R.B. Interações entre o mosaico-em-desenho do feijoeiro e de duas doenças fúngicas, ferrugem e mancha angular, em plantas de feijoeiro. (Dissertação de Mestrado). Piracicaba. Universidade de São Paulo. 1995.

BASSANEZI, R.B., AMORIM,L., BERGAMIN FILHO, A. \& HAU, B. Effects of bean line pattern mosaic virus on the monocyclic components of rust and angular leaf spot of Phaseolus bean at different temperatures. Plant Pathology 47:289-298. 1998.

BERGAMIN FILHO, A. \& AMORIM, L. Doenças de plantas tropicais: epidemiologia e controle econômico. São Paulo: Ceres, 1996.

BUTLER, D.R. \& JADHAV, D.R. Requirements of leaf wetness and temperature for infection of groundnut by rust. Plant Pathology 40:395-400. 1991.

CAMPBELL, C.L. \& MADDEN, L.V. Introduction of plant disease epidemiology. New York: John Wiley, 1990.

CAMPOS, J.A. \& ZAK, L.F. Estudio de algunas características de Isariopsis griseola Sacc., agente causal de la mancha angular del frijol. Agrociencia 39:41-48. 1980.

CARDONA-ALVAREZ, C. \& WALKER, J.C. Angular leaf spot of bean. Phytopathology 46:610-615. 1956.

CARISSE, O. \& KUSHALAPPA, A.C. Development of an infection model for Cercospora carotae on carrot based on temperature and leaf wetness duration. Phytopathology 80:1233-1238. 1990.

CROWE, M.J., COAKLEY, S.M. \& EMGE, R.G. Forecasting dew 
duration at Pendleton, Oregon, using simple weather observations. Journal of Applied Meteorology 17:1482-1487. 1978.

DALLA PRIA, M., BERGAMIN FILHO, A. \& AMORIM, L. Avaliação de diferentes meios de cultura na esporulação de Colletotrichum lindemuthianum, Phaeoisariopsis griseola e Alternaria sp. Summa Phytopathologica 23:188-191. 1997.

GODOY, C. V., CARNEIRO, S. M. T. P. G., IAMAUTI, M. T., DALLA PRIA, M., AMORIM, L., BERGER, R.D. \& BERGAMIN FILHO, A. Diagrammatic scales for bean diseases: development and validation. Zeitschrift fur Planzenkrankheiten und Pflanzenschutz 104:336-345. 1997.

HAU, B. \& KRANZ, J. Mathematics and statistics for analyses in epidemiology. In: Kranz, J. (Ed.) Epidemics of plant disease: mathematics analyses and modeling. Berlin: Springer-Verlag, 1990. pp.12-52.

HAU, B., EISENSMITH, S.P. \& KRANZ, J. Construction of temporal models: II. Simulation of aerial epidemics. In: Gilligan, C.A. (Ed.) Advanced in plant pathology: mathematical modeling of crop disease. London: Academic Press, 1985. v.3, pp.12-52.

HOWARD, A.M. \& GILLESPIE, T.J. A field use guide for surface wetness duration measurement. Guelph: University of Guelph, 1985.

KRANZ, J. \& HAU, B. Systems analysis in epidemiology. Annual Review of Phytopathology 18:67-83. 1980.

KULIK, M.M. Symptomatology and epidemiology of several green bean diseases incited by seed-borne fungi. Seed Science and Technology 12:841-850. 1984.

LLANOS, C.M. Patogenicidad del Isariopsis griseola Sacc. en frijol (Phaseolus vulgaris L.). Acta Agronomica 7:165-170. 1957.

OLAVE, C.A.L. Resistencia de algunas variedades y líneas de frijol (Phaseolus vulgaris L.) al Isariopsis griseola Sacc. Acta Agronômica 8:197-219. 1958.

PARADELA FILHO, O. Algumas moléstias fúngicas do feijoeiro que ocorrem em São Paulo. In: Simpósio Brasileiro do Feijão, 1., Campinas, 1971. 1972. v.2, pp.305-384.

PARLEVLIET, J.E. Partial resistance of barley of leaf rust. Puccinia hordei I. Effect of cultivar and development stage on latent period. Euphytica 24:21-27. 1975.

PENNYPACKER, S.P. \& STEVENSON, R.E. The management of plant pathogens. In: Hatfield, J.L. \& Thomas, I.J. (Eds.).
Biometereology in integrated pest management. London: Academic Press, 1982. pp.243-266.

RAVA, C.A. \& SARTORATO, A. Antracnose. In: Sartorato, A. \& Rava, C.A. (Eds.). Principais doenças do feijoeiro comum e seu controle. Brasília: EMBRAPA,SPI, 1994. pp.17-39.

ROTEM, J. Techniques of controlled-condition experiments. In: Kranz, J. \& Rotem, J. (Eds.). Experimental Techniques in Plant Disease Epidemiology. Berlin: Springer-Verlag, 1988. pp.19-31.

SARTORATO, A. Resistência vertical e horizontal do feijoeiro comum (Phaseolus vulgaris L.) a Isariopsis griseola Sacc. (Tese de Doutorado). Piracicaba. Universidade de São Paulo. 1989.

SARTORATO, A. \& RAVA, C.A. Mancha angular. In: Sartorato, A. \& Rava, C.A. (Eds.). Principais doenças do feijoeiro comum e seu controle. Brasília: EMBRAPA,SPI, 1994. pp.41-68.

SCHUH, W. \& ADAMOWICZ, A. Influence of assessment time and modeling approach on the relationships between temperatureleaf wetness periods and disease parameters of Septoria glycines on soybean. Phytopathology 83:941-948. 1993.

SINGH, A.K. \& SAINI, S.S. Inheritance of resistance to angular leaf spot (Isariopsis griseola Sacc.) in French bean (Phaseolus vulgaris L.). Euphytica 29:175-176. 1980.

STEEL, R.G.D. \& TORRIE, J.H. Principles and procedures of statistic. New York: McGraw Hill, 1980.

SUTTON, J.C., GILlESPIE, T.J. \& HILDBRAND, P.D. Monitoring weather factors in relation to plant disease. Plant Disease 68:78-84. 1984.

VALLAVIEILLE-POPE, C. de, HUBER, L., LECONTE, M. \& GOYEAU, H. Comparative effects of temperature and interrupted wet periods on germination, penetration, and infection of Puccinia recondita $\mathrm{f}$. sp. tritici and $P$. striiformis on wheat seedlings. Phytopathology 85:409-415. 1995.

VENUS, J.C. \& CAUSTON, D.R. Plant growth analysis: the use of Richards function as an alternative to polynomial exponential. Annals of Botany 43:623-632. 1979.

VIEIRA, C. Melhoramento do feijoeiro (Phaseolus vulgaris L.) no Estado de Minas Gerais, IV: estudos realizados no período de 1970 a 1973. Revista Ceres 21:470-485. 1974.

ZADOKS, J.C. \& SCHEIN, R.D. Epidemiology and plant disease management. Oxford: University Press, 1979. 